\title{
Discriminative Analysis-Synthesis Dictionary Learning for Image Classification
}

\author{
Meng Yang ${ }^{13}$, Member, IEEE, Heyou Chang ${ }^{2}$ and Weixin Luo ${ }^{1}$ \\ ${ }^{1}$ College of Computer Science \& Software Enginnering, Shenzhen University, Shenzhen, \\ China \\ ${ }^{2}$ Nanjing University of Science and Technology, Nanjing, P.R. China \\ ${ }^{3}$ School of Data and Computer Science, Sun Yat-Sen University, Guangzhou, China
}

\begin{abstract}
Dictionary learning has played an important role in the success of sparse representation. Although discriminative synthesis dictionary learning for sparse representation with a high-computational-complexity $l_{0}$ or $l_{1}$ norm constrain$\mathrm{t}$ has been well studied for image classification, jointly and discriminatively learning an analysis dictionary and a synthesis dictionary is still in its infant stage. As a dual of synthesis dictionary, the recently developed analysis dictionary can provide a complementary view of data representation, which can have a much lower time complexity than sparse synthesis representation. Although several class-specific analysis-synthesis dictionary, which may have a big correlation between different classes' dictionaries, have been developed, how to learn a more compact and discriminative universal analysis-synthesis dictionary is still open. In this paper, to provide a more complete view of discriminative data representation, we propose a novel model of discriminative analysis-synthesis dictionary learning (DASDL), in which a linear classifier based on the coding coefficient is jointly learned with the dictionary pair, thus the performance of the classifier and the representational power of the dictionary pair being considered at the same time by the same optimization procedure. The size of the learned dictionaries can be very small since the analysis-synthesis dictionary is shared by all class data. An iterative algorith$m$ to efficiently solve the proposed DASDL is presented in this paper. The experiments on face recognition, gender classification, action recognition and
\end{abstract}

Email address: yangmengpolyu@gmail.com, cv_hychang@126.com, 493752864@qq.com ( Meng Yang ${ }^{13}$, Member, IEEE, Heyou Chang ${ }^{2}$ and Weixin Luo $^{1}$ )

Preprint submitted to Neurocomputing

August 15, 2016

(C) 2016. This manuscript version is made available under the Elsevier user license http://www.elsevier.com/open-access/userlicense/1.0/ 
image classification clearly show the superiority of the proposed DASDL.

Keywords: Discriminative, Analysis-Synthesis Dictionary Learning, Image Classification

\section{Introduction}

Inspired by the success of sparse coding in image processing [1, 2] and the discovery of sparsity mechanism in human vision perception [3], sparse representation has been widely applied to a variety of problems, ranging from computer vision to pattern recognition [4]. For example, based on sparse representation, Zhou et al [5] have developed a practical system for fast detection of defects occurring on the surface of bottle caps. As indicated by [6], a dictionary (i.e., a set of representation bases) plays an important role in the success of sparse representation, and learning the desired dictionary from training data instead of using off-the-shelf bases (e.g., wavelets) has led to state-of-the-art results in many practical applications, such as image denoising [1], face recognition [7, 8], and image classification [9, 10].

According to the way of encoding input signals, the dictionary used in sparse representation could be categorized into synthesis dictionary [11] and analysis dictionary [12. Therefore dictionary learning approaches could be mainly divided into three categories: synthesis dictionary learning, analysis dictionary learning, and analysis-synthesis dictionary pair learning.

Synthesis dictionary learning: This kind of dictionary learning methods has been well studied and most of existing dictionary learning methods belong to this category. Traditionally, a linear combination of dictionary atoms is used to synthetically reconstruct a signal. Given the training data $\mathbf{X} \in$ $\mathcal{R}^{m \times n}$, where $n$ is the number of training samples and $m$ is the dimensionality of a training sample, a synthesis dictionary $\mathbf{D}$ could be learned via

$$
\min _{\mathbf{D}, \mathbf{A}}\|\mathbf{X}-\mathbf{D A}\|_{F}^{2}+\Theta(\mathbf{D}, \mathbf{A}) \quad \text { s.t. }\left\|\mathbf{d}_{i}\right\|_{2}^{2}=1 \forall i
$$

where $\mathbf{D}=\left[\mathbf{d}_{1}, \ldots, \mathbf{d}_{k}\right] \in \mathcal{R}^{m \times k}$ is a desired synthesis dictionary, $k$ is the number of dictionary atoms, $\mathbf{A} \in \mathcal{R}^{k \times n}$ is the representation coefficient matrix, and $\Theta(\mathbf{D}, \mathbf{A})$ is a task-driven regularizer (e.g., sparsity of $\mathbf{A}$ ) [13].

A representative unsupervised dictionary learning method is K-SVD [11, which has shown promising performance in image restoration. Then Smith 
and Elad [14] improved K-SVD dictionary learning by modifying the dictionary learning update and sparse coding. Tillmann [15] has studied the computational intractability of exact and approximate dictionary learning. Moreover, jointly learning a dictionary and a nonlinear dimension reduction has also been proposed [16]. For classification tasks, discriminative derivations [7, 8] of K-SVD were developed by jointly learning a dictionary and a classifier based on the coding coefficients. The learned dictionary in [7] is a shared dictionary that could represent data from all classes. The learned synthesis dictionary can be class-specific, in which each dictionary atom has a single class label, e.g., label-consistent K-SVD (LC-KSVD) [8] associated label information with each dictionary atom. Following this line, classspecific dictionary learning, e.g., dictionary learning with structured incoherence (DLSI) [9] and Fisher discrimination dictionary learning (FDDL) [10], were developed by requiring that a class-specific sub-dictionary should properly represent the corresponding class but being weak for the other classes. To fully explore the advantage of class-specific dictionaries and shared dictionaries, some hybrid dictionary learning models (i.e., including both shared and class-specific dictionary atoms) were proposed. Zhou et al. [17] learned a hybrid dictionary with a Fisher-like regularizer on the representation coefficients, while Kong et al. [18] learned a hybrid dictionary by introducing an incoherence penalty term to the class-specific sub-dictionaries.

Although promising results have been reported in the synthesis dictionary learning, the coding coefficients need to be computed by the time-consuming sparse coding. Moreover, synthesis sparse coding cannot give an intuitive illustration like feature transformation (e.g., image operator), so analysis sparse coding, as a dual analysis viewpoint, has been studied recently.

Analysis dictionary learning: Recently, analysis dictionary learning has attracted much attention [12, 19]. The representative analysis dictionary learning approach is the analysis K-SVD [12], which is an unsupervised learning approach. Compared to synthesis dictionary, analysis dictionary representation, i.e., $\mathbf{\Omega X}$, has some unique merits, e.g., generalization to feature transformation and image convolution. Rubinstein et al. [12] proposed to analytically represent the training data (here we denote an analysis dictionary by $\Omega=\left[\mathbf{w}_{1}^{T} ; \ldots ; \mathbf{w}_{k}^{T}\right] \in \mathcal{R}^{k \times m}$ to distinguish it from the synthesis dictionary $\mathrm{D}$, where each row of $\Omega$ is an analysis atom):

$$
\min _{\boldsymbol{\Omega}}\|\boldsymbol{\Omega X}\|_{0}+\Theta(\boldsymbol{\Omega}) \quad \text { s.t. }\left\|\mathbf{w}_{i}\right\|_{2}^{2} \leq 1 \forall i
$$


where $\|\cdot\|_{0}$ counts the number of non-zero entries, and $\Theta(\Omega)$ is a task-driven regularizer (e.g., $\Omega$ can not be a trivial solution). The analysis dictionary, $\Omega$, aims to make $\Omega \mathbf{X}$ a sparse representation coefficient matrix, serving a similar role to $\mathbf{A}$.

Although promising result in image restoration has been reported in [12], analysis K-SVD is still computationally complicated and not designed for classification task due to the lack of enough discrimination.

Analysis-synthesis dictionary pair learning: Synthesis dictionary represents an input signal by using a linear combination of dictionary atoms, while analysis dictionary directly transforms a signal to a sparse feature space by multiplying the signal, which provides a complementary view of data representation. In the view of computation, synthesis dictionary representation involves a high-time-complexity sparse coding, while analysis-synthesis dictionary representation can reconstruct a signal with analysis coding coefficient, which can be fast computed by a linear projection. To provide a faster and more complete view of data representation, analysis-synthesis dictionary learning has been proposed. Rubinstein and Elad [20] proposed an analysis-synthesis dictionary learning (ASDL) model for the task of image processing. In ASDL, a pair of synthesis dictionary and analysis dictionary was learned from an image patch set to pursue their good representation ability and sparsity of coding coefficients. Different from ASDL, Gu et al. 21] proposed a projective dictionary pair learning (PDPL) for image classification. In PDPL, the synthesis class-specific dictionary and analysis classspecific dictionary were required to well represent the corresponding class but poorly represent the other classes. For classification tasks, obviously ASDL ignores to introduce discrimination into the learned dictionaries. Although PDPL has introduced the class-specific structure into dictionary pair learning, it learned a pair of dictionaries for each class, resulting in high redundancy between different class-specific dictionaries and large size of the learned dictionary. How to learn a more compact and discriminative universal analysis-synthesis dictionary is still open.

In this paper, we propose a novel model of discriminative analysis-synthesis dictionary learning (DASDL). In the proposed model, a classifier based on the coding coefficient is jointly learned with the dictionary pair. Incorporating the classification stage directly into the dictionary-learning procedure can gain a discriminative power for the dictionary. For the coding coefficient, a thresholding operator is used to make it sparse and a regression residual of its projection to its label space is minimized to embed discrimination. 
The proposed DASDL is evaluated on the application of face recognition, gender classification, action recognition and image classification. Compared with other state-of-the-art dictionary learning methods, DASDL has better or competitive performance in various classification tasks.

In our paper, the number of training samples, the number of classes, the number of dictionary atoms, and the dimensionality of signal are denoted by $n, c, k$ and $m$, respectively. We denote matrices and vectors by bold-italic capital letters and lowercase letters, respectively. The rest of this paper is organized as follows. Section 2 briefly introduces the related work. Section 3 presents the proposed DASDL model. Section 4 conducts experiments, and Section 5 concludes the paper.

\section{Related work}

Rubinstein and Elad [20] proposed an analysis-synthesis dictionary learning (ASDL) for image deblurring. ASDL explicitly learned a pair of analysis dictionary and synthesis dictionary via

$$
\min _{\boldsymbol{\Omega}, \mathbf{D}, \boldsymbol{\lambda}}\left\|\mathbf{X}-\mathbf{D} S_{\boldsymbol{\lambda}}(\boldsymbol{\Omega X})\right\|_{F}^{2} \quad \text { s.t. }\left\|\mathbf{w}_{i}\right\|_{2}=1 \forall i
$$

where $\boldsymbol{\Omega}=\left[\mathbf{w}_{1}^{T} ; \ldots ; \mathbf{w}_{k}^{T}\right]$ is an analysis dictionary, $\mathbf{w}_{i} \in \mathcal{R}^{m}, \mathbf{D}=\left[\mathbf{d}_{1}, \ldots, \mathbf{d}_{k}\right]$ is a synthesis dictionary, $\mathbf{d}_{i} \in \mathcal{R}^{m}, \boldsymbol{\lambda}=\left[\lambda_{1}, \ldots, \lambda_{k}\right]$ and $\lambda_{i}$ is the threshold for the $i$-th row of $\Omega . S_{\boldsymbol{\lambda}}(\cdot)$ is a hard thresholding function operating on matrices with $k$ rows, thresholding the $i$-row by $\lambda_{i}$ (i.e., $\forall j$, if $\left|[\boldsymbol{\Omega X}]_{i, j}\right| \geq$ $\lambda_{i},\left[S_{\lambda_{i}}(\boldsymbol{\Omega X})\right]_{i, j}=[\boldsymbol{\Omega X}]_{i, j}$; otherwise, $\left.\left[S_{\lambda_{i}}(\boldsymbol{\Omega X})\right]_{i, j}=0\right)$. The $l_{2}$ norm of $\mathbf{d}_{i}$ or $\mathbf{w}_{i}$ is usually required to be unity in order to avoid a trivial solution. It is easy to see that in ASDL, the learned dictionary pair is only required to well represent the training samples, without considering its application to classification task.

Following ASDL, Gu et al.[21] proposed a projective dictionary pair learning (PDPL) for pattern classification. The core idea of PDPL is that both analysis and synthesis dictionaries are class-specific dictionaries. Defining $\mathbf{D}_{c}$ and $\boldsymbol{\Omega}_{c}$ as the $c$ th-class synthesis sub-dictionary and analysis sub-dictionary respectively, PDPL can be formulated as

$$
\left.\min _{\boldsymbol{\Omega}, \mathbf{D}} \sum_{c=1}^{C} \| \mathbf{X}_{c}-\mathbf{D}_{c} \boldsymbol{\Omega}_{c} \mathbf{X}_{c}\right)\left\|_{F}^{2}+\lambda\right\| \boldsymbol{\Omega}_{c} \overline{\mathbf{X}}_{\mathbf{c}} \|_{F}^{2}
$$


where $\overline{\mathbf{X}}_{\mathbf{c}}$ is the training data of all classes except class $c$. Eq. (4) requires the class-specific dictionary pair of class $c$ (e.g. $\mathbf{D}_{c}$ and $\boldsymbol{\Omega}_{c}$ ) well represent the $c$-th training data but poorly represent the training data of $\overline{\mathbf{X}}_{\mathbf{c}}$. This structured dictionary regularization in PDPL makes the learned dictionary pair discriminative for classification task, and promising results have been presented in [21].

With the theoretical development of synthesis and analysis dictionary learning, dictionary learning based sparse representation has been widely applied to image denoising [1], face recognition [7, 8], image classification [9, 10] and detection [5]. For example, Song et al [22] adopted sparse error correction (SEC) model to minimize the energy of the errors and proposed SEC-DKSVD for iris recognition. By introducing Fisher criterion, Zheng et al 23] proposed Fisher discriminative KSVD (FD-KSVD) to learn an over complete discriminative dictionary and an optimal linear classifier for image classification and Dong et al [24] proposed a supervised dictionary learning method for action recognition in still images. Our proposed discriminative analysis-synthesis dictionary learning can also be applied to various image classification tasks, such as face recognition, gender classification, scene categorization.

\section{Discriminative Analysis-Synthesis Dictionary Learning}

Although PDPL has introduced the class-specific structure into dictionary pair learning, its possible large dictionary size and high redundancy between different classes can be detrimental to the classification performance in terms of time complexity and discrimination ability. In contrast, ASDL can learn a small size dictionary pair, but no discrimination information is introduced to make it discriminative for classification. The above problems may be overcome if we can learn a smaller-sized dictionary from the given training images while maintaining the discriminative power of the dictionary. In this section, we propose to learn a discriminative analysis-synthesis dictionary with a smaller size that can not only well represent data from all classes but also have a powerful discrimination for image classification tasks. In this section, we first present the discriminative term, and then describe the proposed discriminative analysis-synthesis dictionary model and the optimization of DASDL under two different situations (i.e. predefined threshold and learning threshold), the classification strategy is presented at the end of this section. 


\subsection{Discriminative Term for Coding Coefficients}

As in [7, 8], we aim to include the classification error as a term in the objective function for dictionary learning to make the dictionary optimal for classification. Here, we use a linear predictive classifier. The classification error which is based on the coding coefficient is designed as:

$$
\left\|\mathbf{Y}-\mathbf{P} S_{\boldsymbol{\lambda}}(\boldsymbol{\Omega X})\right\|_{F}^{2} \quad \text { s.t. }\|\mathbf{P}\|_{F}^{2} \leq \sigma
$$

where $\mathbf{P}=\left[\mathbf{p}_{1}, \ldots, \mathbf{p}_{k}\right] \in \mathcal{R}^{C \times k}$ is a projection matrix, and $\mathbf{Y}=\left[\mathbf{Y}_{1}, \ldots, \mathbf{Y}_{C}\right] \in$ $\mathcal{R}^{c \times n}$ is a label matrix with $n=\sum_{c} n_{c}$, where $n_{c}$ is the number of training samples in $c$-th class. $Y_{c}$ is a label sub-matrix of class $c$, which is defined as:

$$
c^{\text {th }} \text { row }\left[\begin{array}{cccc}
0 & 0 & \cdots & 0 \\
\vdots & \vdots & 0 & \vdots \\
1 & 1 & \cdots & 1 \\
\vdots & \vdots & 0 & \vdots \\
0 & 0 & \cdots & 0
\end{array}\right]
$$

The discriminative term of Eq.(5) aims to make the coding coefficient, $S_{\boldsymbol{\lambda}}(\Omega \mathbf{X})$, discriminative via projecting the $c$-th class coding coefficients only to $c$-th dimension of the label space.

\subsection{DASDL Model}

By jointly considering the representation ability and the discrimination ability of the analysis-synthesis dictionary, the proposed discriminative analysissynthesis dictionary learning model can be represented as

$$
\begin{aligned}
& \min _{\boldsymbol{\Omega}, \mathbf{P}, \mathbf{D}, \boldsymbol{\lambda}}\left\{\left\|\mathbf{X}-\mathbf{D} S_{\boldsymbol{\lambda}}(\boldsymbol{\Omega X})\right\|_{F}^{2}+\gamma\left\|\mathbf{Y}-\mathbf{P} S_{\boldsymbol{\lambda}}(\boldsymbol{\Omega X})\right\|_{F}^{2}\right\} \\
& \text { s.t. }\left\|\mathbf{d}_{i}\right\|_{2}=1, \forall i ; \quad\|\mathbf{P}\|_{F}^{2} \leq \sigma
\end{aligned}
$$

where $\gamma$ is a scalar parameter, the first term can ensure that the learned dictionary pair of $\mathbf{D}$ and $\boldsymbol{\Omega}$ well represents the training data, and the second term is to make sure that the coding coefficient generated by the analysis dictionary could have strong discrimination ability.

\subsection{Solving Algorithm of DASDL Model}

In order to learn the pair of synthesis dictionary and analysis dictionary efficiently, we adopt the protocol in the original K-SVD algorithm and refor- 
mulate problem (6) as

$$
\begin{aligned}
& \min _{\boldsymbol{\Omega}, \mathbf{P}, \mathbf{D}, \boldsymbol{\lambda}}\left\{\left\|(\mathbf{X} ; \sqrt{\gamma \mathbf{Y}})-(\mathbf{D} ; \sqrt{\gamma} \mathbf{P}) S_{\boldsymbol{\lambda}}(\boldsymbol{\Omega X})\right\|_{F}^{2}\right. \\
& \text { s.t. }\left\|\mathbf{d}_{i}\right\|_{2}=1, \forall i ; \quad\|\mathbf{P}\|_{F}^{2} \leq \sigma
\end{aligned}
$$

Let $\mathbf{X}_{n e w}=(\mathbf{X} ; \sqrt{\gamma} \mathbf{Y}), \mathbf{D}_{n e w}=(\mathbf{D} ; \sqrt{\gamma} \mathbf{P})$, and $\mathbf{D}_{n e w}$ be normalized column-wise. Therefor, we can further drop the regularization penalty term $\|\mathbf{P}\|_{F}^{2} \leq \sigma$. The optimization of $(7)$ is equivalent to solving the following problem:

$$
\min _{\boldsymbol{\Omega}, \mathbf{D}_{\text {new }}, \boldsymbol{\lambda}}\left\{\left\|\mathbf{X}_{\text {new }}-\mathbf{D}_{\text {new }} S_{\boldsymbol{\lambda}}(\boldsymbol{\Omega X})\right\|_{F}^{2} \quad \text { s.t. } \quad\left\|\mathbf{d}_{\text {new }, i}\right\|_{2}=1, \forall i\right.
$$

\subsubsection{Solving $\boldsymbol{\Omega}$ and $\mathbf{D}_{\text {new }}$ with predefined threshold}

Since threshold $\boldsymbol{\lambda}$ is predefined, we focus on the updating of $\boldsymbol{\Omega}$ and $\mathbf{D}_{\text {new }}$. Here, we simply set $\lambda_{1}=\lambda_{2}=\ldots=\lambda_{k}$, problem (8) can be rewritten as:

$$
\min _{\boldsymbol{\Omega}, \mathbf{D}_{n e w}}\left\{\left\|\mathbf{X}_{n e w}-\mathbf{D}_{n e w} S_{\boldsymbol{\lambda}}(\boldsymbol{\Omega X})\right\|_{F}^{2} \quad \text { s.t. } \quad\left\|\mathbf{d}_{n e w, i}\right\|_{2}=1, \forall i\right.
$$

The problem of (9) can be efficiently solved by updating the dictionary pair atom by atom. At the $i$-th step, we keep all but the $i$-th pair of atoms fixed. After isolating the dependence on the $i$-th atom pair, we can rewrite problem (9) as

$$
\min _{\mathbf{w}_{i}, \mathbf{d}_{\text {new }, i}}\left\{\left\|\mathbf{E}_{i}-\mathbf{d}_{\text {new }, i} S_{\boldsymbol{\lambda}}\left(\mathbf{w}_{i}^{T} \mathbf{X}\right)\right\|_{F}^{2} \quad \text { s.t. } \quad\left\|\mathbf{d}_{\text {new }, i}\right\|_{2}=1\right.
$$

where $\mathbf{E}_{i}=\mathbf{X}_{n e w}-\sum_{j \neq i} \mathbf{d}_{n e w, j} S_{\boldsymbol{\lambda}}\left(\mathbf{w}_{j}^{T} \mathbf{X}\right)$. The problem of Eq. 10, which has a very similar formation to Eq.(11) of [20], could be efficiently solved using the rank-one approximation algorithm in [20].

Let $S_{\boldsymbol{\lambda}}($.$) define a partition of \boldsymbol{X}$ into two sets: $J$ and $\bar{J}$, with current $\boldsymbol{w}_{i}$, where $S_{\boldsymbol{\lambda}}\left(\boldsymbol{w}_{i}^{T} \boldsymbol{X}^{J}\right)=\boldsymbol{w}_{i}^{T} \boldsymbol{X}^{J}$ and $S_{\boldsymbol{\lambda}}\left(\boldsymbol{w}_{i}^{T} \boldsymbol{X}^{\bar{J}}\right)=\mathbf{0}$. $\boldsymbol{E}_{j}$ is similarly spitted to the submatrices $\boldsymbol{E}_{j}^{J}$ and $\boldsymbol{E}_{j}^{\bar{J}}$. Then we approximate Eq. 10 to

$$
\min _{\mathbf{w}_{i}, \mathbf{d}_{\text {new }, i}}\left\{\left\|\mathbf{E}_{i}^{J}-\mathbf{d}_{n e w, i} \mathbf{w}_{i}^{T} \mathbf{X}^{J}\right\|_{F}^{2} \quad \text { s.t. } \quad\left\|\mathbf{d}_{n e w, i}\right\|_{2}=1\right.
$$

Inspired by the rank-one approximation algorithm in [20], the algorithm of updating the $i$-th atom pair $\left\{\mathbf{w}_{i}, \mathbf{d}_{n e w, i}\right\}$ with predefined $\boldsymbol{\lambda}$ is summarized in Alg. 1. 


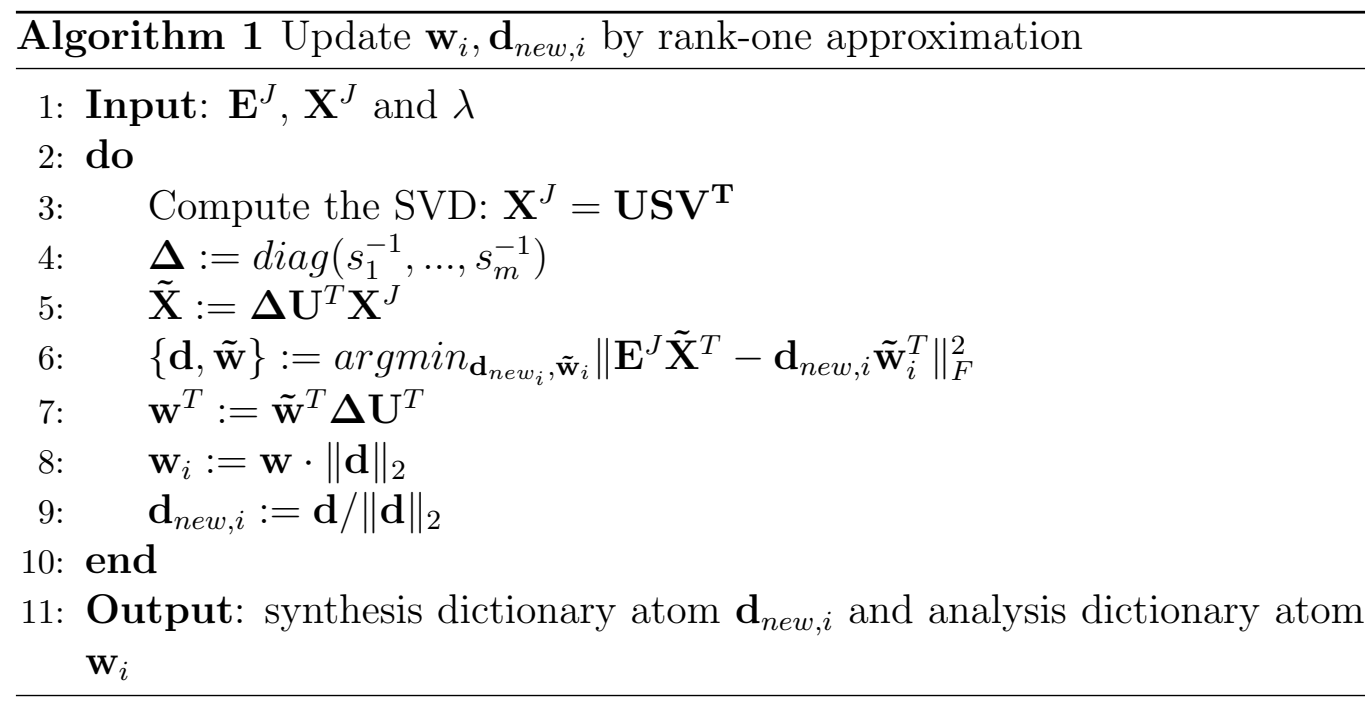

\subsubsection{Solving $\boldsymbol{\Omega}, \mathbf{D}_{\text {new }}$ with learning threshold}

In this situation, we optimization (8) using a sequential approach similar to the K-SVD [11]. At the $i$-th step, we first update the $i$-th dictionary atom pair $\left\{\mathbf{w}_{i}, \mathbf{d}_{\text {new }, i}\right\}$ via Alg.(1) by replacing $\lambda$ with $\lambda_{i}$, then update $\lambda_{i}$ to match the new atoms. The problem in (8) translates to the following optimization task:

$$
\min _{\lambda_{i}}\left\|\mathbf{E}_{i}-\mathbf{d}_{n e w, i} S_{\lambda_{i}}\left(\mathbf{w}_{i}^{T} \mathbf{X}\right)\right\|_{F}^{2}
$$

As [20], this problem can be globally solved due to the discrete nature of the hard threshold operator. Suppose that all signals are ordered such that $\left|\mathbf{w}_{i}^{T} \mathbf{x}_{1}\right| \leq\left|\mathbf{w}_{i}^{T} \mathbf{x}_{2}\right| \leq \cdots \leq \mathbf{w}_{i}^{T} \mathbf{x}_{n} \mid$. For any threshold $\lambda_{i} \in\left(\left|\mathbf{w}_{i}^{T} \mathbf{x}_{1}\right|,\left|\mathbf{w}_{i}^{T} \mathbf{x}_{n}\right|\right)$, there exists a unique index $\kappa=\kappa\left(\lambda_{i}\right)$ such that $\left|\mathbf{w}_{i}^{T} \mathbf{y}_{\kappa-1 \mid}<\lambda_{i} \leq\right| \mathbf{w}_{i}^{T} \mathbf{y}_{\kappa} \mid$. Then we can rewrite 12 as:

$$
\min _{\lambda_{i}} \sum_{j=1}^{\kappa\left(\lambda_{i}\right)-1}\left\|\mathbf{e}_{j}\right\|_{2}^{2}+\sum_{i=\kappa\left(\lambda_{i}\right)}^{n}\left\|\mathbf{e}_{j}-\mathbf{d}_{n e w, i} \mathbf{w}_{i}^{T} \mathbf{x}_{j}\right\|_{2}^{2}
$$

where $\mathbf{e}_{j}$ is the $j$-th column of $\mathbf{E}_{i} . \lambda_{i}$ can be solved once $\kappa$ is solved. Since $\kappa$ is a discrete point $(\kappa \in\{1, \ldots, n\})$, the optimization of $(13)$ can carried out over all $k$ and take the global minimum. The threshold update process is summarized in Alg. 2.

The whole solving algorithm of DASDL is summarized in Alg $3 . \mathbf{D}_{\text {new }}$ could be initialized as the dictionary learned from $\mathbf{X}_{n e w}$ via K-SVD [11], and 


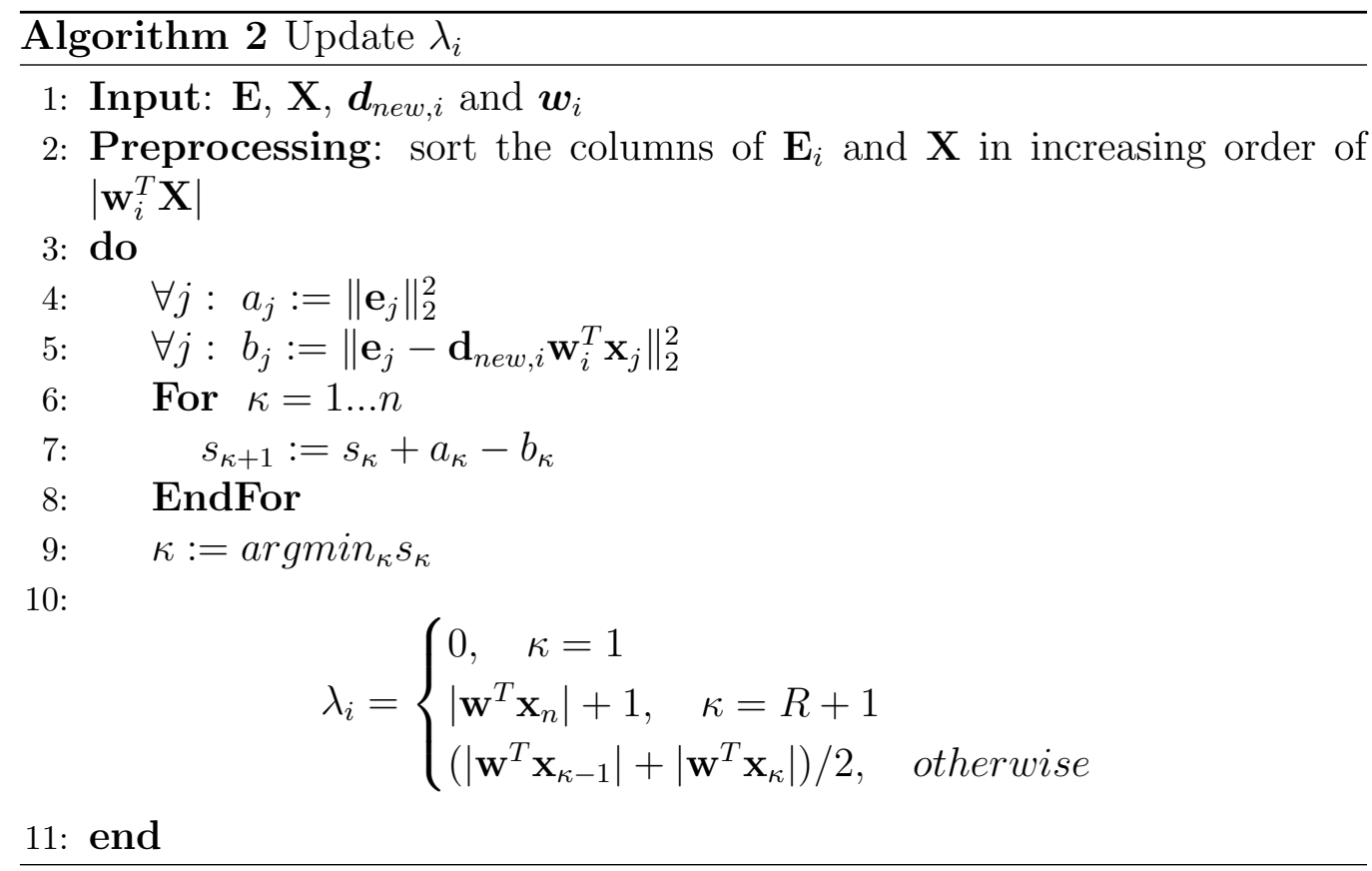

$\boldsymbol{\Omega}$ is initialized as $\left(\mathbf{D}_{\text {new }}^{T} \mathbf{D}_{\text {new }}\right)^{-1} \mathbf{D}_{\text {new }}^{T} \mathbf{X}_{\text {new }} \mathbf{X}$, which is the solution of Eq.(8) without consider $S_{\boldsymbol{\lambda}}(\cdot)$. A convergence curve of the solving algorithm on gender classification of AR [25] dataset is given in the Fig.1. It could be seen that the DASDL algorithm could converge to a satisfactory local optimal solution.

\subsubsection{Classification Strategy}

When the algorithm in Alg. 3 is finished, we can obtain a synthesis dictionary $\mathbf{D}$, an analysis dictionary $\boldsymbol{\Omega}$, a threshold vector $\boldsymbol{\lambda}$ and a projection matrix $\mathbf{P}$. However, we cannot simply use $\mathbf{P}$ for testing, since $\mathbf{D}$ and $\mathbf{P}$ are normalized jointly in the previous learning algorithm, i.e., $\left\|\mathbf{d}_{i} ; \sqrt{\gamma} \mathbf{p}_{i}\right\|_{2}=1$. We should re-normalize the dictionary atoms and the projection matrix $\mathbf{P}$ because we require that $\left\|\mathbf{d}_{i}\right\|_{2}=1$ in Eq.(6). Since $\mathbf{D}$ is not used in the classification phase, we only normalize the projection matrix via

$$
\mathbf{p}_{i}=\mathbf{p}_{i} /\left\|\mathbf{d}_{i}\right\|_{2}^{2}
$$

For a test sample $\mathbf{y}$, we first compute its sparse representation $\mathbf{z}=$ $S_{\boldsymbol{\lambda}}\left(\boldsymbol{\Omega}^{T} \mathbf{y}\right)$. Then we simply use the linear projective matrix $\mathbf{P}$ to estimate 


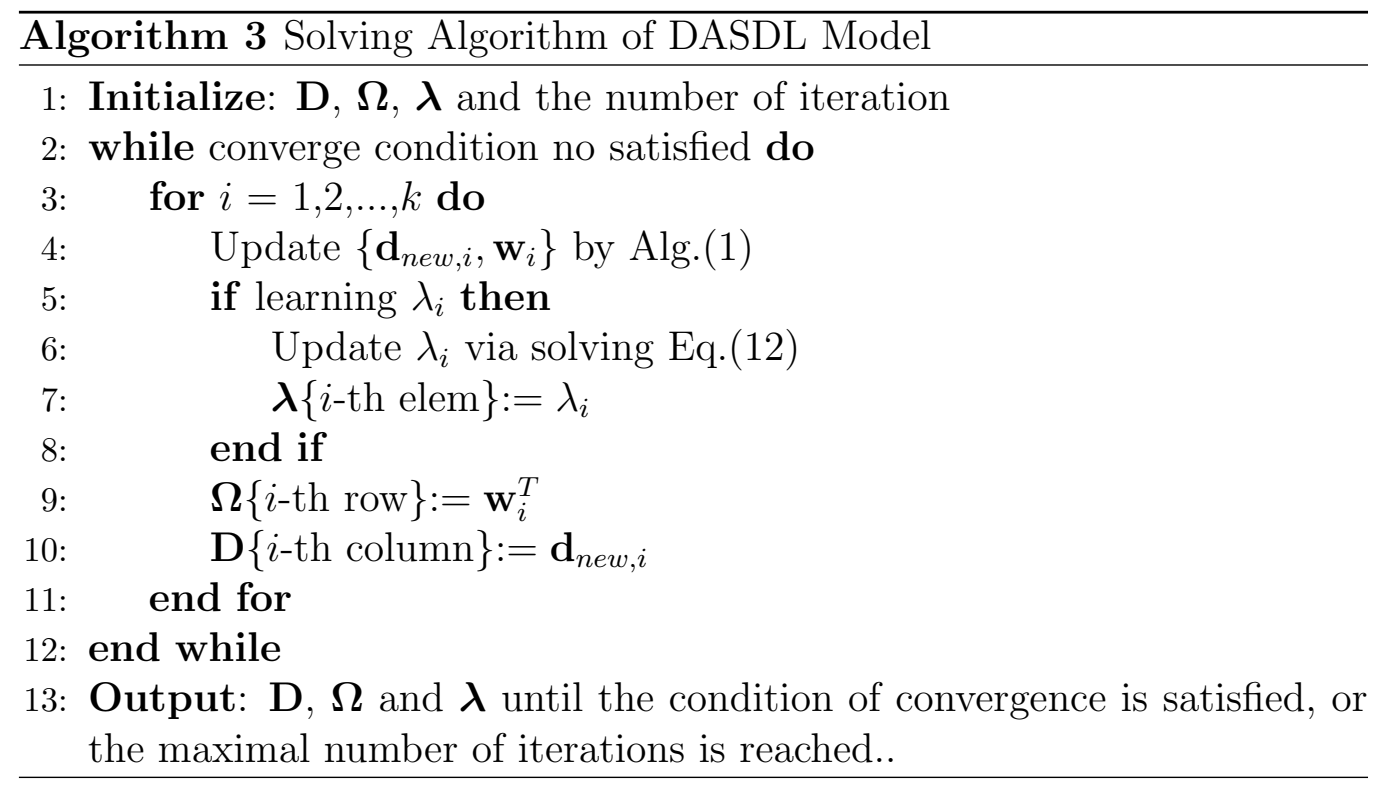

a label vector $\boldsymbol{l}=\mathbf{P z}=\left[l_{1}, \ldots, l_{C}\right]$. The label of $\mathbf{y}$ is given by

$$
i d e n t i t y(\mathbf{y})=\operatorname{argmax}_{i}\left\{l_{i}\right\}, \quad \text { s.t. } \boldsymbol{l}=\mathbf{P z}
$$

\section{Experimental Results}

We performed experiments on face recognition, gender classification, action recognition and image classification to demonstrate the performance of DASDL. We evaluated DASDL on the applications of face recognition, gender classification, action recognition and image classification in Section 4.1, Section 4.2, Section 4.3, and Section 4.4 respectively.

To clearly illustrate the advantage of DASDL, we compared it with several latest dictionary learning methods, such as Discriminative K-SVD (DKSVD) [11, Label Consistent K-SVD (LC-KSVD) [8], analysis-synthesis dictionary learning with SVM as a classifier (ASDL-SVM) [20], Fisher discrimination dictionary learning (FDDL) [10, and the projective dictionary pair learning (PDPL) [21]. Besides, we also report sparse representation based classifier (SRC) 4 and some methods for special tasks.

The number of dictionary atom is an important factor on the final performance. In order to make a fair comparison, we set the number of dictionary 


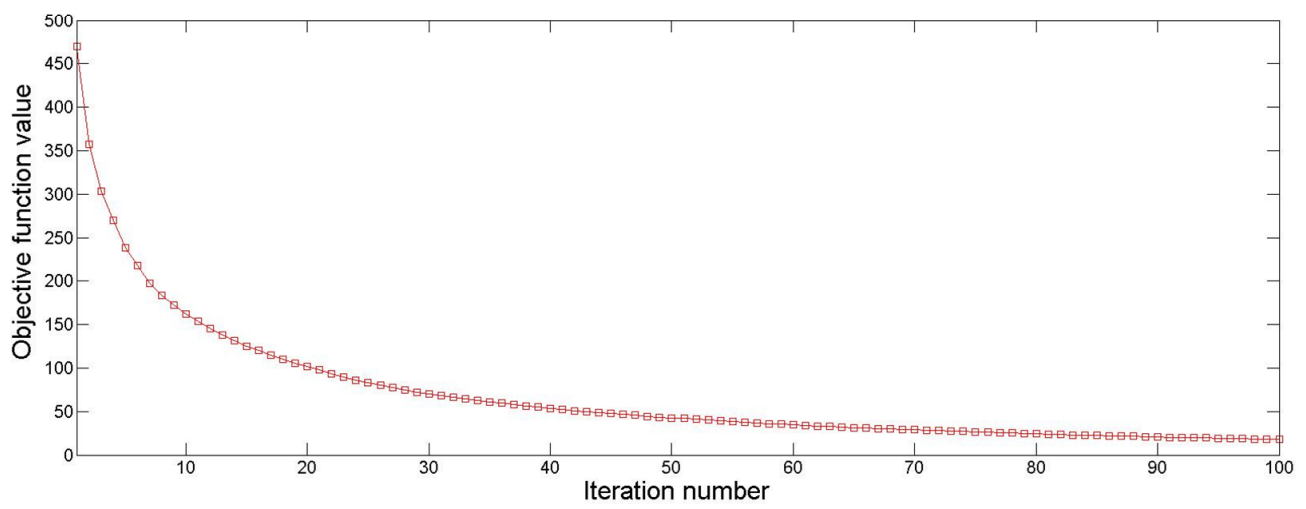

Figure 1: The convergence of the solving algorithm with learning threshold in the gender classification on AR database.

atoms for all competing methods as 100 (i.e, $k=100$ ) in face recognition, gender classification and action recognition. The dictionary size is set as 20, 100, 102 and 257 on the Cifar10, Cifar100, Caltech101 and Caltech256, respectively . The synthesis dictionary $\mathbf{D}_{n e w}$ is initialized on whole training data $\mathbf{X}_{\text {new }}$. Once $\mathbf{D}_{\text {new }}$ is initialized, the analysis dictionary $\boldsymbol{\Omega}$ is initialized by $\boldsymbol{\Omega}=\left(\mathbf{D}_{\text {new }}^{T} \mathbf{D}_{\text {new }}\right)^{-1} \mathbf{D}_{\text {new }}^{T} \mathbf{X}_{\text {new }} \mathbf{X}^{-1}$, followed by a normalization on each row of $\boldsymbol{\Omega}$. There are two parameters in the proposed DASDL. $\gamma$ makes a balance between the representation term and the discriminative term. $\lambda$ controls the sparsity of the coding coefficients. In our paper, parameter $\gamma$ is fixed as 0.005. If parameter $\lambda$ is predefined, $\lambda$ is fixed as $1 e-5$.

\subsection{Face Recognition}

AR face database [25], is used to evaluate the performance of the proposed DASDL. The AR dataset contains illumination, expression, and disguise variation. Following the experimental setting of AR in [8], a set of 2,600 images of 50 females and 50 males subjects are extracted. Some samples of male and female are shown in Fig.(2). For each person, we randomly select 20 images for training and the other 6 for testing. 540-d feature (i.e., same to randomface [4] by using a random dimensionality-reduction projection) provided by [8] is used as the facial features. We repeated the experiment 3 times with different random spits of the training and testing images to obtain reliable results. The final recognition rates are reported as the average of each run.

We compared the proposed DASDL with other competing methods and the experimental results were listed in Table 1. The accuracy of DASDL 


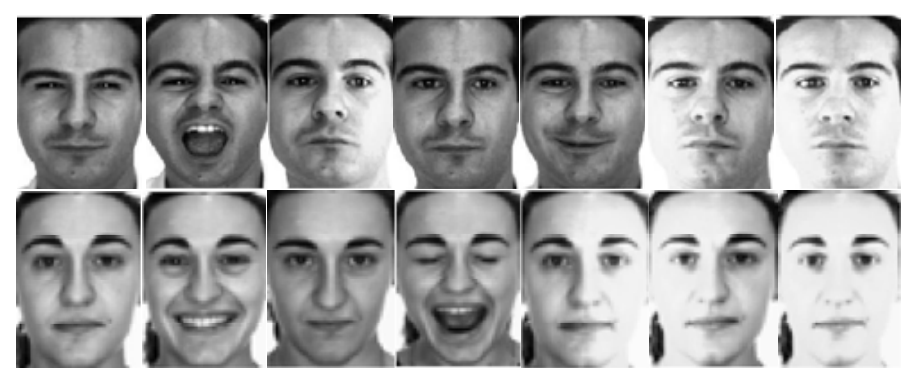

Figure 2: Some samples of male and female from AR database.

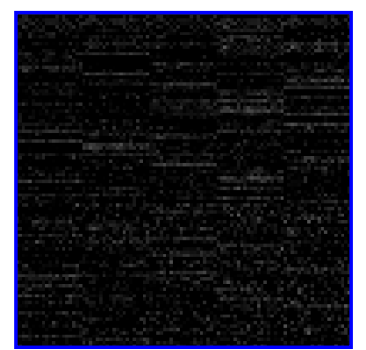

$\operatorname{DASDL}_{p}$

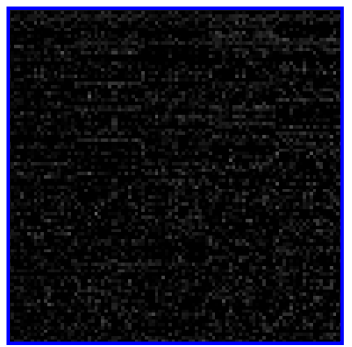

ASDL

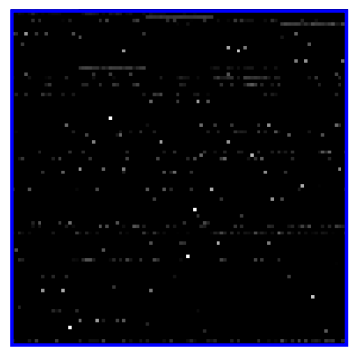

LCKSVD

Figure 3: Comparison of representation for training samples from the first five classes on the AR dataset. Here we normalize the coefficients associated to each dictionary atom to make a better visualization

with predefined threshold is denoted as $\mathbf{D A S D L} \mathbf{S}_{p}$, the accuracy with learning threshold is denoted as $\mathbf{D A S D L} l$. In this dataset, DASDL achieves the best performance, e.g., $96.3 \%$ accuracy, and has over $1 \%$ improvements over all the other competing methods. Compared to ASDL-SVM, the improvement of DASDL is over 4\%. It is surprising that both $\mathrm{SRC}^{*}$ and FDDL have a low recognition rate which may be because only one dictionary atom could be used for each class. Compared to $\mathrm{SRC}^{*}$ and FDDL, the learned dictionary atoms in DASDL have the discriminative and powerful representation ability because they are required to represent all class data with discriminative coefficients. For DASDL, the accuracy with predefined threshold is the same with that with learning threshold.

We visualize the representation of $\mathrm{DASDL}_{p}$, ASDL and LC-KSVD for the first five classes, shown as Figure 3. The representation generate a classspecific structure in our method, which is absent in ASDL and LC-KSVD. 
Table 1: Face Recognition Accuracy (\%) on AR Database

\begin{tabular}{cc|cc}
\hline \hline Method & Accuracy & Method & Accuracy \\
\hline ASDL-SVM & 91.8 & LC-KSVD & 73.8 \\
DKSVD & 89.2 & PDPL & 95.2 \\
SRC $^{*}$ & 59.8 & DASDL $_{p}$ & $\mathbf{9 6 . 3}$ \\
FDDL & 68.3 & DASDL $_{l}$ & $\mathbf{9 6 . 3}$ \\
\hline \hline
\end{tabular}

\subsection{Gender Classification}

In this experiment we chose a non-occluded subset (14 images per subject) of AR consisting of 50 male subjects and 50 female subjects. Images of the first 25 males and 25 females were used for training, and images of the remaining 25 males and 25 females for testing. The size of image is resized from $165 \times 120$ to $60 \times 43$. We used $\mathrm{PCA}$ to reduce the dimension of each image to 100 .

The experimental results of all the competing methods are shown in Table 2. It can be seen that DASDL is visibly better than all the other methods. The accuracy of DASDL is not only at least 3\% higher than DKSVD and LC-KSVD, but also at least 1.5\% higher than ASDL-SVM and PDPL. Compared to ASDL-SVM that separately encodes data and trains a classifier, DASDL jointly learns discriminative coding coefficients and a powerful classifier. Both PDPL and FDDL learn two class-specific dictionaries for male and female data, respectively. However, due to the large intra-class variation in each gender data, the class-specific dictionary may not have enough discriminative representation ability. In contrary, DASDL, which requires the coding coefficient associated to the shared dictionary should be discriminative, can better handle this task. FDDL is the second best method with $1.2 \%$ gap compared to DASDL; however, the testing time of FDDL (i.e., 2.27 second) is much larger than that of DASDL $(0.02$ second $)$. As for DASDL, the accuracy with learning threshold has faint advantage over that with learning threshold.

\subsection{Action Recognition}

The benchmark action dataset, UCF sports action dataset [26], is used to conduct the action classification experiment. The dataset collected video clips from various broadcast sports channels (e.g., BBC and ESPN). The 
Table 2: Gender Classification Accuracy (\%) on AR Database

\begin{tabular}{cc|cc}
\hline \hline Method & Accuracy & Method & Accuracy \\
\hline ASDL-SVM & 88.1 & LC-KSVD & 84.4 \\
DKSVD & 86.6 & PDPL & 86.0 \\
SRC* & 59.1 & DASDL $_{p}$ & $\mathbf{8 9 . 6}$ \\
FDDL & 88.4 & DASDL $_{l}$ & $\mathbf{9 0 . 3}$ \\
\hline \hline & & & \\
\hline
\end{tabular}

Figure 4: Some samples of actions in UCF sports action dataset.

action bank features of 140 videos provided by [27] are adopted in the experiment. The provided action bank feature, which includes a large set of individual action detectors at varying scales and view points, is a high-level descriptor for video activity recognition. These videos cover 10 sport action classes: driving, golfing, kicking, lifting, horse riding, running, skateboarding, swinging-(prommel horse and floor), swinging-(high bar) and walking, some of which are shown in Fig. 4.

As the experiment setting in [8], we evaluated the DASDL via five-fold cross validation and the average result of five tests is reported. Here the dimension of the action bank feature is reduced to 100 via PCA, and the performance of some specific methods for action recognition, such as sparse dictionary-based representation [16], action back feature with SVM classifier [18] are also reported in Table 3. From Table 3, we can see that DASDL still has higher recognition rate compared to the traditional dictionary learning methods and some specific approaches. This further validates that the joint learning of discriminative dictionary and classifier is powerful. DASDL outperforms PDPL and ASDL by $1.5 \%$ and 5\%, respectively. Although both DASDL and FDDL all achieve the best performance, DASDL $(\mathbf{0 . 0 0 6 5}$ second) is about 100 times faster than FDDL (0.6032 second) since there is a time consuming sparse coding in FDDL. 
Table 3: Action Recognition Accuracy (\%) on the UCF Sports Action Database

\begin{tabular}{cc|cc}
\hline \hline Method & Accuracy & Method & Accuracy \\
\hline$[28]$ & 83.6 & KSVD & 86.8 \\
{$[27]$} & 90.7 & FDDL & $\mathbf{9 3 . 6}$ \\
ASDL-SVM $^{*}$ & 88.6 & PDPL & 92.1 \\
SRC $^{*}$ & 92.9 & DASDL $_{p}$ & $\mathbf{9 3 . 6}$ \\
DKSVD $_{\text {LC-KSVD }}^{88.1}$ & DASDL $_{l}$ & $\mathbf{9 3 . 6}$ \\
\hline \hline
\end{tabular}

\subsection{Image Classification}

The Caltech-101 dataset [29] contains 9,144 images from 102 classes (i.e., 101 object classes and a 'background' class) including animals, vehicles, flowers, and so on. The samples from each category have significant shape variability. The number of images in each category varies from 31 to 800 . Compared to the Caltech-101 dataset, the Caltech-256 dataset 30] contains 30,607 images of 257 categories. There are at least 80 images per category. Following the common experimental settings, we randomly select 30 samples per category for training and the rest for testing on these two datasets. The dictionary size is set as 102 and 257 on the two datasets, respectively. The CIFAR-10 dataset [31] consists of 60,000 $32 \times 32$ color images in 10 classes, with 6000 images per class. The dataset is divided into five training batches and one test batch, each with 10000 images. The test batch contains exactly 1000 randomly-selected images for each class. The training batches contain the remaining images in random order. Here we randomly choose 1000 samples per class in all five training batches for training, and the test batch for testing. Just like the Cifar-10 dataset, the Cifar-100 dataset [31] has 100 classes containing 600 images each. There are 500 training (training batch) and 100 testing (test batch) images per class. some of which are shown in Fig. 5. In the experiment, we randomly choose 100 samples per class in training batch for training and test batch for testing.

The feature of each image on the Caltech-101 and Cifar-10 dataset is extracted by LSAE [32]. The image feature on the Caltech-256 and Cifar100 dataset is extracted as [33], where the SIFT descriptors [34] are extracted densely from each image on a grid with stepsize 6 pixels, followed by learning a dictionary with 1024 atoms, and lastly the SPM feature [35] is computed 


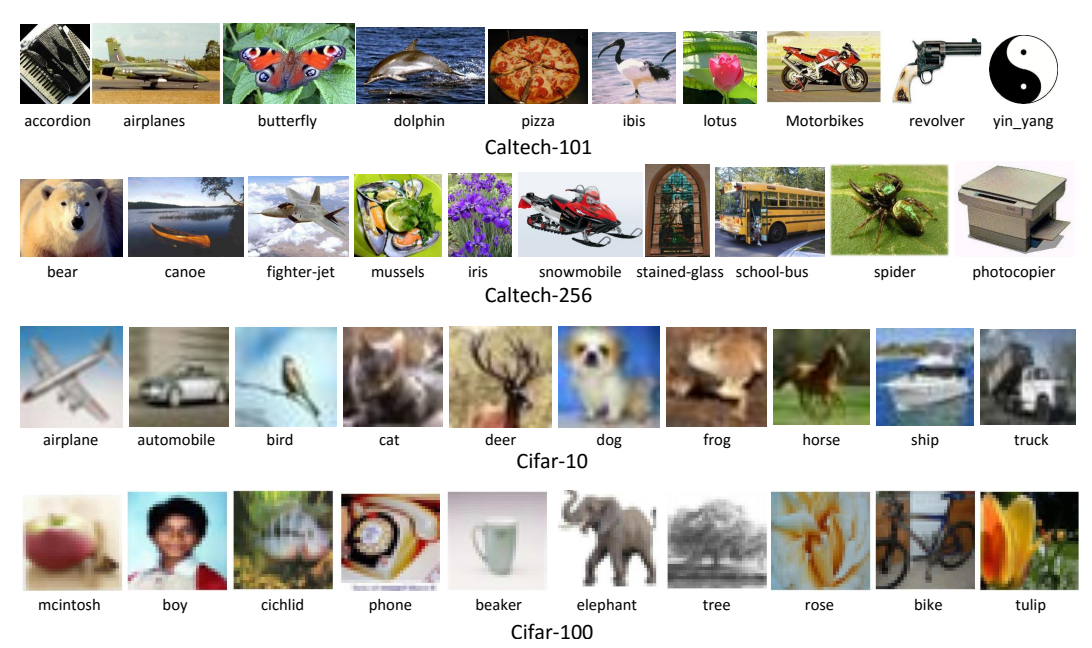

Figure 5: Some samples on the Caltech-101, Caltech-256, Cifar-10 and Cifar100 .

for each image by setting the spatial block structure as $1 \times 1,2 \times 2$ and $4 \times 4$. Then the features are reduced to 500 dimension by PCA on the four datasets. The parameter $\lambda$ and $\gamma$ are set as 0.75 and 0.0005 respectively in the four datasets. We repeat the experiments 3 times with different random spits of the training and testing images to obtain reliable results. The final recognition rates are reported as the average of each run, show as Table 4.

It can be seen that DASDL achieves better performance than all the other methods. In particular, DASDL outperforms PDPL by $0.63 \%, 0.13 \%, 2.56 \%$ and $0.87 \%$ on the four datasets respectively. Under the MATLAB R2014a programming environment and in a CPU server of $16 \mathrm{CPUs}$ with $1.3 \mathrm{GHZ}$ and 16G RAM, the training and testing time of different methods on the four datasets under is shown as Table. 5. From Table. 5, we can see that DASDL is faster than ASDL-SVM and FDDL when training, but slower than other four methods due to the computation of SVD. Nevertheless, the efficiency of online testing is more important than that of the offline training. As for testing, DASDL is very effective, the time-consuming of DASDL is only more than LC-KSVD on Caltech-101 and Caltech-256, and on Cifar-10 and Cifar100 datasets, DASDL is only slower than LC-KSVD and PDPL. Although the accuracy of DASDL is less than $1 \%$ higher than FDDL, the second best accuracy, DASDL is about 1000 times faster than FDDL $(0.0002 \mathrm{~s}$ vs $1.21 \mathrm{~s}$ on the Caltech-101 dataset, $0.0007 \mathrm{~s}$ vs $1.51 \mathrm{~s}$ on the Caltech-256 dataset, 
Table 4: Image Classification Accuracy (\%) on the Caltech-101, Caltech-256, Cifar-10 and Cifar-100

\begin{tabular}{c|cccc}
\hline \hline & Caltech-101 & Caltech-256 & Cifar-10 & Cifar-100 \\
\hline DKSVD & 64.38 & 23.29 & 45.60 & 11.61 \\
LC-KSVD & 65.72 & 24.48 & 45.76 & 13.83 \\
SRC & 46.49 & 11.62 & 28.08 & 5.43 \\
ASDL-SVM & 71.25 & 33.83 & 54.73 & 19.36 \\
FDDL & 75.16 & 34.08 & 60.08 & 20.18 \\
PDPL & 74.84 & 33.99 & 58.62 & 19.57 \\
DASDL $_{p}$ & $\mathbf{7 5 . 5 4}$ & $\mathbf{3 4 . 1 2}$ & $\mathbf{6 1 . 1 8}$ & $\mathbf{2 0 . 4 4}$ \\
DASDL $_{l}$ & $\mathbf{7 5 . 4 7}$ & $\mathbf{3 4 . 2 1}$ & $\mathbf{6 1 . 8 7}$ & $\mathbf{2 0 . 5 9}$ \\
\hline \hline
\end{tabular}

Table 5: Training time (Testing time)(s) on the Caltech-101, Caltech-256, Cifar-10 and Cifar-100

\begin{tabular}{l|cccc}
\hline \hline & Caltech-101 & Caltech-256 & Cifar-10 & Cifar-100 \\
\hline DKSVD & $395.4(8.3 \mathrm{e}-3)$ & $1354.2(1.1 \mathrm{e}-2)$ & $166.4(5.9 \mathrm{e}-4)$ & $1407.8(8.2 \mathrm{e}-3)$ \\
LC-KSVD & $20.2(3.3 \mathrm{e}-5)$ & $163.2(3 \mathrm{e}-4)$ & $10.7(4 \mathrm{e}-5)$ & $60.5(1.7 \mathrm{e}-4)$ \\
SRC & $0(2.1 \mathrm{e}-2)$ & $0(4.3 \mathrm{e}-2)$ & $0(2.8 \mathrm{e}-2)$ & $0(5.7 \mathrm{e}-2)$ \\
ASDL-SVM & $2155.8(1.1 \mathrm{e}-3)$ & $31246.5(2.3 \mathrm{e}-2)$ & $1205.0(4 \mathrm{e}-4)$ & $6274.8(2.8 \mathrm{e}-3)$ \\
FDDL & $5610.7(1.21)$ & $128364.8(1.51)$ & $854.4(1 \mathrm{e}-1)$ & $24631.7(8.3 \mathrm{e}-1)$ \\
PDPL & $6.9(6 \mathrm{e}-4)$ & $36.8(7 \mathrm{e}-4)$ & $2.3(2 \mathrm{e}-5)$ & $10.9(3.8 \mathrm{e}-4)$ \\
DASDL $_{p}$ & $515.5(2 \mathrm{e}-4)$ & $5731.6(6 \mathrm{e}-4)$ & $551.1(1.1 \mathrm{e}-4)$ & $5331.7(7.1 \mathrm{e}-4)$ \\
DASDL $_{l}$ & $517.3(2 \mathrm{e}-4)$ & $5754.8(6 \mathrm{e}-4)$ & $554.0(1.1 \mathrm{e}-4)$ & $5373.4(7.1 \mathrm{e}-4)$ \\
\hline \hline
\end{tabular}

$0.0001 \mathrm{~s}$ vs $0.099 \mathrm{~s}$ on the Cifar- 10 dataset and $0.0007 \mathrm{~s}$ vs $0.83 \mathrm{~s}$ on the Cifar- 100 dataset). The proposed DASDL has achieved the best accuracy with a very fast testing speed, which is because a shared and discriminative dictionary is jointly learned with a classifier. Meanwhile, in the testing phase, only two matrix and vector multiplications are needed to do classification, which makes the testing very fast.

\section{Conclusion}

This paper presented a novel discriminative analysis-synthesis dictionary learning model for image classification. It has been shown that the discrimination of the learned dictionary pair could be exploited via jointly learning 
a linear classifier based on the coding coefficient. Since the proposed DASDL is a shared dictionary for all classes, the size of DASDL could be very small while with a good performance. We also proposed an efficient solving algorithm for the proposed model. The proposed DASDL method was extensively evaluated on several image classification tasks. The experimental results clearly demonstrated that DASDL outperforms or is comparable to previous state-of-the-art methods, such as FDDL, DKSVD, ASDL, LCKSVD and PDPL.

Compared to prevailing discriminative dictionary learning, the proposed DASDL not only provides a more complete view of data representation, but also generate a discriminative small-size dictionary pair, on which the analysis coding coefficients can be efficiently solved in the testing phase. One open problem still in the proposed DASDL is the efficiency of dictionary pair learning in DASDL since singular-value-decomposition (SVD) is involved in the optimization of DASDL. In the future, we will avoid to use SVD and develop a more efficient dictionary pair learning algorithm of DASDL.

\section{Acknowledgments}

This work is partially supported by the National Natural Science Foundation for Young Scientists of China (Grant no. 61402289), National Science Foundation of Guangdong Province (Grant no. 2014A030313558), and Shenzhen Scientific Research and Development Funding Program (Grant no. JCYJ20140509172609171), Scientific Research Fund for new teacher of Shenzhen University (Grant no. 201536), Scientific Research fund for Advanced Talents of Shenzhen University (Grant no. 000070).

\section{References}

[1] M. Elad, M. Aharon, Image denoising via sparse and redundant representations over learned dictionaries, IEEE Trans on Image Processing 15 (12) (2006) 3736-3745.

[2] Y. M. J.C. Yang, J. Wright, T. Huang, Image super-resolution as sparse representation of raw image patches, in: CVPR, 2008.

[3] B. A. Olshausen, D. J. Field, Emergence of simple-cell receptive field properties by learning a sparse code for natural images, Letters to Nature 381 (13). 
[4] J. Wright, A. Y. Yang, A. Ganesh, Y. Ma, Robust face recognition via sparse representation, IEEE Transactions on Pattern Analysis and Machine Intelligence 31 (2) (2009) 210-227.

[5] W. Zhou, M. Fei, H. Zhou, K. Li, A sparse representation based fast detection method for surface defect detection of bottle caps, Neuralcomputation 123 (2015) 406-414.

[6] A. B. R. Rubinstein, M. Elad, Dictionaries for sparse representation modeling, Proceedings of the IEEE 98 (6) (2010) 1045-1057.

[7] Q. Zhang, B. Li, Discriminative k-svd for dictionary learning in face recognition, in: CVPR, 2010.

[8] Z. Jiang, Z. Lin, L. S. Davis, Label consistent k-svd: Learning a discriminative dictionary for recognition, IEEE Transactions on Pattern Analysis and Machine Intelligence 35 (11) (2013) 2651-2664.

[9] I. Ramirez, P. Sprechmann, G. Sapiro, Classification and clustering via dictionary learning with structured incoherence and shared features, in: CVPR, 2010.

[10] M. Yang, L. Zhang, X. Feng, D. Zhang, Fisher discrimination dictionary learning for sparse representation, in: ICCV, 2011.

[11] M. Aharon, M. Elad, A. Bruchstein, K-svd:an algorithm for designing overcomplete dictionaries for sparse representation, IEEE Transactions on Signal Processing 54 (11) (2006) 4311-4322.

[12] T. P. R. Rubinstein, M. Elad, Analysis k-svd: A dictionary-learning algorithm for the analysis sparse model, IEEE Trans on Signal Processing 61 (3) (2013) 661-677.

[13] J. Marial, F. Bach, J. Ponce, Task-driven dictionary learning, IEEE Transactions on Pattern Analysis and Machine Intelligence 34 (4) (2012) 791-804.

[14] L. Smith, M. Elad, Improving dictionary learning: Multiple dictionary updates and coefficient reuse, IEEE Signal Processing Letters 20 (1) (2013) 79-82. 
[15] A. Tillmann, On the computational intractability of exact and approximate dictionary learning, IEEE Signal Processing Letters 22 (1) (2015) $45-49$.

[16] K. B. Y. Zhou, Locality constrained dictionary learning for nonlinear dimensionality reduction, IEEE Signal Processing Letters 20 (4) (2013) 335-338.

[17] N. Zhou, Y. Shen, J. Peng, J. Fan, Learning inter-related visual dictionary for object recognition, in: CVPR, 2012.

[18] S. Kong, D. Wang, Learning inter-related visual dictionary for object recognition, in: ECCV, 2012.

[19] Z. C. Zhanglin.Peng, Ya Li, L. Lin, Deep boosting: Joint feature selection and analysis dictionary learning in hierarchy, in: arXiv:1508.01887v1, 2014.

[20] R.Rubinstein, M. Elad, Dictionary learning for analysis-synthesis thresholding, IEEE Trans on Signal Processing 62 (22) (2014) 5962 5972 .

[21] W. Z. S. Gu, L. Zhang, X. Feng, Projective dictionary pair learning for pattern classification, in: NIPS, 2014.

[22] Y.Song, W.Can, Z.He, Robust iris recognition using sparse error correction model and discriminative dictionary learning, Neuralcomputation 137 (2014) 198-204.

[23] H.Zheng, D.Tao, Discriminative dictionary learnig via fisher discriminative k-svd algorithm, Neuralcomputation 162 (2015) 9-15.

[24] J. Dong, C. Sun, W. Yang, A supervised dictionary learning and discriminative weighting model for action recognition, Neuralcomputation 158 (2015) 246-256.

[25] A. Martinez, R. Benavente, The ar face database, Tech. rep., CVC Tech. Report (1998).

[26] M. Rodriguez, J. Ahmed, M. Shah, A spatio-temporal maximum average correlation height filter for action recognition, in: CVPR, 2008. 
[27] S. Sadanand, J. Corso, Action bank: A high-level representation of activity in video, in: CVPR, 2012.

[28] Z. J. Q. Qiu, R. Chellappa, Sparse dictionary-based representation and recognition of action attributes, in: ICCV, 2011.

[29] F. Li, R.Fergus, P.Perona, Learning generative visual models from few training samples: An incremental bayesian approach tested on 101 object categories, in: ICCV Workshop gennerative model based vision, 2004.

[30] G.Griffin, A.Holub, P.Perona, Caltech-256 object category data set, Tech. rep., CIT Tech. Report (2007).

[31] A. Krizhevsky, Learning multiple layers of features from tiny images, Tech. rep., Univ. Toronto Tech. Report (2009).

[32] W. Luo, J. Yang, W. Xu, T. Fu, Locality-constrained sparse autoencoder for image classification, IEEE Signal Processing Letters 22 (8) (2015) 1070-1073.

[33] J. Yang, K. Yu, Y. Gong, T. Huang, Linear spatial pyramid matching using sparse coding for image classification, in: CVPR, 2009.

[34] D. G. Lowe, Object recognition from local scale-invariant features, in: ICCV, 1999.

[35] S. Lazebnik, C. Schmid, J. Ponce, Beyond bags of features: spatial pyramid matching for recognizing natural scene categories, in: CVPR, 2006. 